\section{How eukaryotes express themselves: Short and sweet}

\author{
Gene Regulation - A Eukaryotic Perspective \\ (4th edn) \\ D Latchman \\ Nelson Thornes Ltd, UK; 2003. 323 pp. \\ $£ 27.00$, paperback. ISBN 0-7487-6530-1
}

Heredity (2003) 91, 5. doi:10.1038/sj.hdy.6800272

Reviewed by PJ Scotting

The first edition of this book set out to provide a text with introductory sections appropriate for second-year undergraduates, and more advanced material for finalyear undergraduates and researchers or clinicians new to the field. Now in its 4th edn, these aims seem well satisfied by the books content. The text progresses from a well-balanced and concise review of basic transcriptional and translational mechanisms to more detailed consideration of control at the levels of transcription, posttranscription and chromatin structure. The final chapters cover a more detailed discussion of the DNA elements and proteins involved in transcriptional control and finally the relation between gene expression and human disease. The written style is very easy to read and the organisation logical. In particular, explanations of key experimental approaches used by those who first discovered aspects of gene regulation provide the reader with a real idea of how we got where we are with this topic. The 4th edn aims to update previous editions with attention to those areas that have developed most over recent years. To that end, there is a more detailed description of transcriptional repression and brief mention of the latest methods to carry out global analyses of gene expression such as arrays and proteomics.

The book does have some weaknesses. The figures are kept admirably simple, but they could have been made rather more 'interesting' and perhaps clearer, using three-dimensional representation of structures (as opposed to the flat-line drawings used) even if the same limited colour reproduction were retained. The book would also be improved by a more thorough crossindexing. Currently, it can require a significant level of prior knowledge to find relevant material. With respect to transcription factors, it is a little disappointing that the text tends to cover the usual suspects. Our understanding of huge numbers of new factors and families of factors has expanded rapidly over the past 10 years, but many of these are not even mentioned. Clearly, a detailed description of all transcription factors would be beyond the scope of a book at this level. However, one is left with the impression that all of the major transcription factor classes have been mentioned. The book would certainly benefit from some kind of review of the plethora of transcription factors, their DNA binding domains, etc. This could readily be presented in tabular form. Along with this, reference to reviews of these other factors would help to serve this purpose. Currently, the lack of their inclusion provides the reader with no clues and no means to access further information.

Overall, this does indeed provide a text both accessible and suitably informative for undergraduates. Despite the few negative points described above, at $£ 27$, this book is a good candidate for core text that students might actually read as opposed to some competitor texts that can realistically only be used for reference. As for scientists and clinicians, it actually provides a very valuable source of information on basic processes but again does not provide sufficiently comprehensive information or referencing in some areas. In summary, one of the best books in its field, worth buying if you like your information concise and don't care too much for pretty illustrations. As the great 20th century philosopher Homer Simpson might have said 'just because donuts have holes in them, it don't mean they're not tasty'.

PJ Scotting

Children's Brain Tumour Research Centre Institute of Genetics University of Nottingham, QMC Nottingham NG7 2UH, UK

E-mail: paul.scotting@nottingham.ac.uk 